\title{
Novel deletion of the E3A ubiquitin protein ligase gene detected by multiplex ligation-dependent probe amplification in a patient with Angelman syndrome
}

\author{
Francesco Cali ${ }^{1}$, Alda Ragalmuto', \\ Valeria Chiavetta', Giuseppe Calabrese ${ }^{2}$, \\ Marco Fichera ${ }^{3}$, Mirella Vinci ${ }^{1,4}$, Giuseppa Ruggeri ${ }^{1}$, \\ Pietro Schinocca', Maurizio Sturnio ${ }^{3}$, \\ Salvatore Romano ${ }^{2}$, Valentino Romano ${ }^{1,5,6}$ \\ and Maurizio Elia ${ }^{2}$ \\ 'Laboratorio di Genetica Molecolare \\ ${ }^{2}$ U.O. di Neurologia e Neurofisiopatologia Clinica e Strumentale \\ ${ }^{3}$ Laboratorio di Diagnosi Genetica \\ IRCCS Oasi Maria SS \\ Troina (EN), Italy \\ ${ }^{4}$ Dipartimento di Oncologia Sperimentale e Applicazioni Cliniche \\ ${ }^{5}$ Dipartimento di Fisica e Tecnologie Relative, \\ Bdg. 18 Viale delle Scienze \\ Università degli Studi di Palermo \\ Palermo, Italy \\ ${ }^{6}$ Corresponding author: Tel, 39-091-23899067; \\ Fax, 39-091-23860815; E-mail, vromano@unipa.it \\ DOI 10.3858/emm.2010.42.12.087
}

Accepted 4 November 2010

Available Online 11 November 2010

Abbreviations: AS, angelman syndrome; CMDA, comparative multiplex dosage analysis; MLPA, multiplex ligation-dependent probe amplification; RPA, relative peak area; UBE3A, ubiquitin protein ligase $\mathrm{E} 3 \mathrm{~A}$

\begin{abstract}
Angelman syndrome (AS) is a severe neurobehavioural disorder caused by failure of expression of the maternal copy of the imprinted domain located on 15q11-q13. There are different mechanisms leading to AS: maternal microdeletion, uniparental disomy, defects in a putative imprinting centre, mutations of the E3 ubiquitin protein ligase (UBE3A) gene. However, some of suspected cases of AS are still scored negative to all the latter mutations. Recently, it has been shown that a proportion of negative cases bear large deletions overlapping one or more exons of the UBE3A gene. These deletions are difficult to detect by conventional gene-scanning methods due to the masking effect by the non-deleted allele. In this study, we have used for the first time multiplex ligation-dependent probe am-
\end{abstract}

plification (MLPA) and comparative multiplex dosage analysis (CMDA) to search for large deletions affecting the UBE3A gene. Using this approach, we identified a novel causative deletion involving exon 8 in an affected sibling. Based on our results, we propose the use of MLPA as a fast, accurate and inexpensive test to detect large deletions in the UBE3A gene in a small but significant percentage of AS patients.

Keywords: Angelman syndrome; gene dosage; genetic association studies; ubiquitin-protein ligases

\section{Introduction}

Angelman syndrome (AS) (MIM 105830) is a severe neurodevelopmental disorder, whose incidence is estimated to be $1 / 10,000-1 / 20,000$ (Petersen et al., 1995; Clayton-Smith and Laan, 2003). Affected subjects show developmental delay, mental retardation, delayed motor development, movement and balance disorder, gait ataxia, jerky limb movements, epilepsy with abnormal EEG, microcephaly, characteristic facial phenotype, hypopigmentation and scoliosis and absence of speech, a characteristic behavioural profile that includes a happy affect (Robb et al., 1989). Jiang et al. (1999) were among the first to suggest that this phenotype could be caused by the loss of function of one or more normally active maternally-inherited genes on chromosome 15q11-q13. To date, four genetic mechanisms are known to be responsible for AS and include: (i) maternallyderived interstitial deletions (ca. $4 \mathrm{Mb}$ ) of 15q11q13 (70-75\% of cases); (ii) paternal uniparental disomy (UPD) of the whole chromosome 15 (2-5\%); (iii) defects in the imprinting process (ICP) (3-5\%); (iv) nucleotide substitutions as well as small insertion/deletions of the gene encoding E6AP-E3 ubiquitin protein ligase (UBE3A). All these abnormalities always involve a region of chromosome 15, comprising the UBE3A gene, suggesting that a dysfunctional or absent UBE3A protein is a major cause of AS (Kishino et al., 1997; Matsuura et al., 1997; Rougeulle et al., 1997; Abaied et al., 2010).

Chromosomal, molecular and clinical data on AS patients have also been used to attempt a cor- 
Table 1. Molecular genetics analyses performed in 31 subjects with Angelman Syndrome

\begin{tabular}{cccccc}
\hline $\begin{array}{c}\text { Large common } \\
\text { deletions }(4-10 \mathrm{Mb})\end{array}$ & UPD & ICP* & $\begin{array}{c}\text { UBE3A mutation detected } \\
\text { by sequencing }\end{array}$ & $\begin{array}{c}\text { UBE3A mutation detected } \\
\text { by MLPA (this study) }\end{array}$ & Negative \\
\hline $61.3 \%(19)$ & $9.7 \%(3)$ & $3.2 \%(1)$ & $3.2 \%(1)$ & $3.2 \%(1)$ & $19.4 \%(6)$ \\
\hline
\end{tabular}

${ }^{*}$ ICP mutations analysis were not performed. The single patient, positive to methylation test and negative to UPD or deletion and UPD.

relation between genotype/karyotype and the phenotype. Interestingly, it was found that patients carrying large deletions generally exhibit a more severe phenotype while patients with UBE3A mutations are less severely affected (Moncla et al., 1999) and those with uniparental disomy have

A
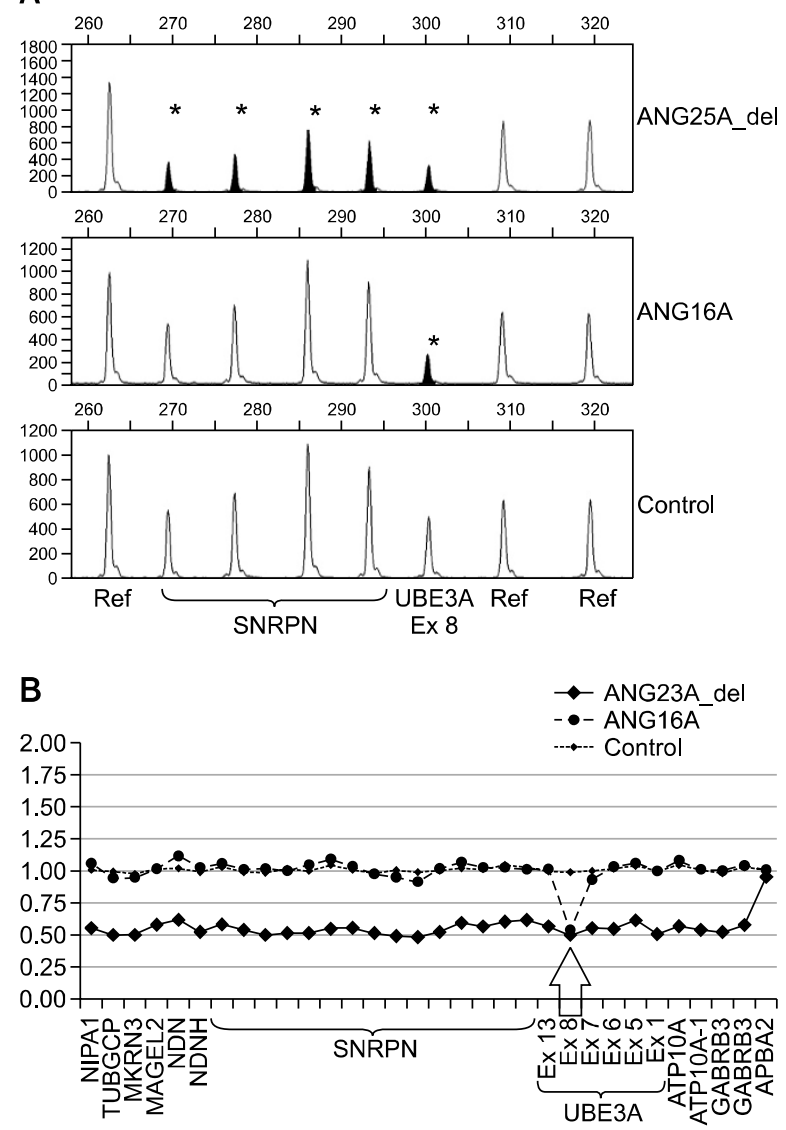

Figure 1. MLPA analysis of the UBE3A gene performed with the SALSA MLPA KIT ME028-B1. (MRC-Holland, Amsterdam, The Netherlands) in a patient (ANG16A) with Angelman syndrome. In this patient the MLPA revealed the deletion of exon 8 . (A) The electropherograms show results referring to only 8 of 53 probes obtained for (i) patient ANG16A, (ii) patient ANG25A_Del (positive control bearing a deletion of $5.7 \mathrm{Mb}$ ), and (iii) a negative control ("Control"). The asterisks above peaks indicate the deleted exons; (B) Graph displaying the ratios between the ANG16A patient's and controls' Relative Peak Areas (RPA) determined in the 15q11-q13 region. Reference values are as follows: RPA Ratio $\sim 1=$ normal dosage; RPA Ratio $\sim 0.5=$ Heterozygous deletion. See text for more details. better verbal development compared to patients with a deletion (Fridman et al., 2000).

Point mutations and small insertions/deletions of the UBE3A gene can be detected with conventional gene scanning methods (e.g., DNA sequence analysis). Large deletions have been rarely reported (Burger et al., 2002; Boyes et al., 2006). However, the impact of these deletions in AS may have been underestimated since they are difficult to detect by conventional gene-scanning methods due to the masking effect by the nondeleted allele. To overcome this limitation, in this study, we have used Multiplex Ligation-dependent Probe Amplification (MLPA) (Schouten et al., 2002) to screen for large disease-causing deletions/duplications of the UBE3A gene. In particular, we have tested 31 AS families whose mutant UBE3A genotype had remained unexplained in our previous methylation and sequence analyses. The use of MLPA led to the identification of a novel deletion of the UBE3A gene in a family.

\section{Results and Discussion}

As suggested by recent evidences, intragenic deletions and duplications may represent common alterations in many clinically diagnosed patients scoring negative to traditional genetic tests $(\mathrm{Ha}$ verfield et al., 2009). Based on these observations we have developed a gene dosage method for the UBE3A gene to genotype patients strictly fulfilling consensus diagnostic criteria for AS (Williams et al., 1995), and negative to MS-PCR methylation and sequencing standard analyses. Table 1 shows our screening data performed in 31 patients with AS phenotype. As shown, $77 \%$ of our patients have mutations which can be identified using conventional mutation analysis. The remaining 7 patients were analysed by MLPA ME028-B1 (MRC-Holland, Amsterdam, The Netherlands) dosage methodology for detection of deletions or duplications of the UBE3A gene exons (Exons \# 1 $5,6,7,8,13$ ) alongside appropriate positive and negative controls for comparison. One patient (ANG16A) showed an altered pattern of amplification compatible with a novel exon 8 deletion of the UBE3A gene (see details on figure 1). 
Analysis of unaffected ANG16A parental samples showed the deletion was also present in the mother while a normal amplification pattern was detected in the father and in both unaffected sisters. Since the MLPA ME028-B1 includes five of ten coding UBE3A exons, we developed a new MLPA-based kit able to detect deletions in the remaining coding exons (Exons \# 9, 10, 11, 12, 14) so to verify whether the deletion detected in the ANG16A patient would possibly extend beyond exon 8. The sensitivity of this test to detect deletion/duplication was verified by comparing the RPA between ten normal individuals (negative controls) and two patients bearing a deletion or a duplication respectively (positive controls) (see figure 2). As shown in this figure, MLPA analyses performed in the ANG16A patient, indicates that no deletion occurred in the other UBE3A's exons. During the preparation of this manuscript, a new commercial kit (P336-A1 UBE3A) was made available by MRC Holland. This kit can detect large deletions/duplications of all UBE3A gene exons. We have reanalyzed patient ANG16A and his mother with this new kit and have confirmed the deletion of exon 8 (data not shown).

In line with hundreds previous MLPA studies performed in various syndromes and diseases (Ainsworth et al., 2004; Madrigal et al., 2007; Priolo et al., 2008), we confirm that MLPA is an useful method to detect UBE3A exon deletions which otherwise would be missed using conventional gene-scanning methods. However, at the same time we need to claim about some limitations in the performance of MLPA for quantification analysis possibly leading to false positives as documented in our previous study (Calì et al., 2010). These observations thus make indispensable the use of other gene dosage analysis methods to confirm the preliminary MLPA findings. For this reason, we developed an alternative semi-quantitative CMDA dosage, PCR-based, method that confirmed the deletion involving UBE3A exon 8 in the patient ANG16A (see the figure 3 ) and in his mother (ANG16M). The MLPA results were also confirmed by long-range PCR using intronic primers hybridizing at both sites of the presumed exon 8 deletion. In patient ANG16A and in his mother (ANG16M) Long-range-PCR successfully amplified a fragment of ca $5 \mathrm{~Kb}$, while the expected $15 \mathrm{~kb}$ normal fragment was obtained in his father and sisters, as well as in a control sample (Figure 4A). By DNA sequence analyses we found that the breakpoints between intron 7 and intron 8 corresponds to a deletion of $9495 \mathrm{bp}$, including the $145 \mathrm{bp}$ of the whole exon 8 (Figure 4). This deletion creates a frameshift altering the aminoacid
A

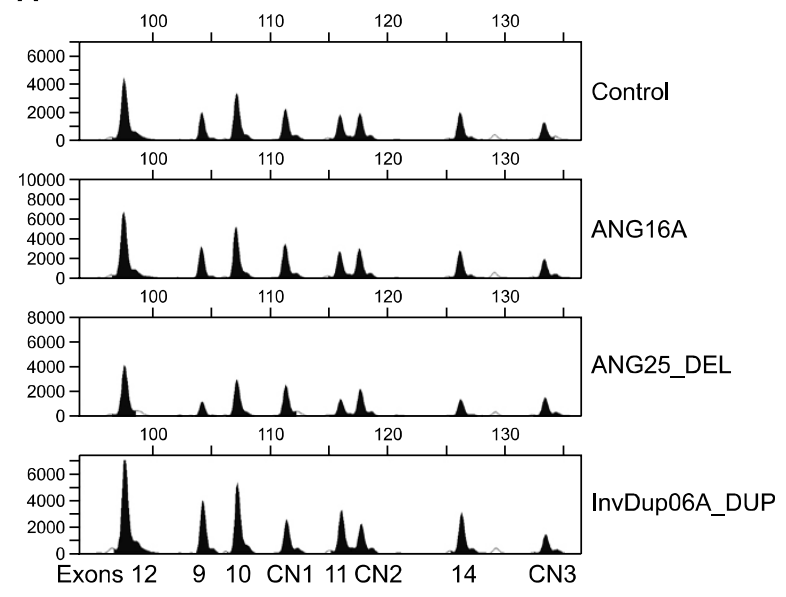

B

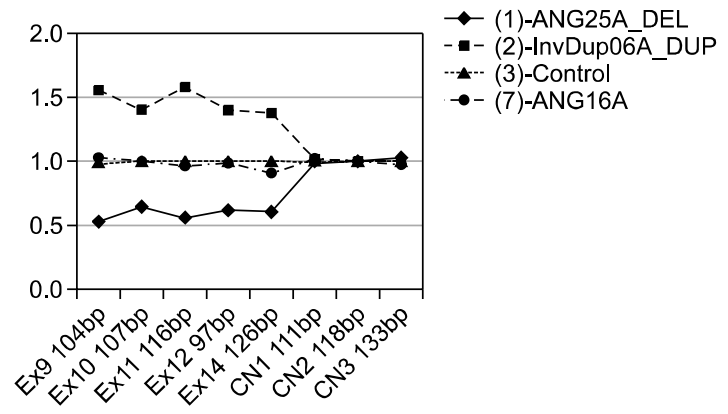

Figure 2. MLPA analysis performed using a kit, developed in our laboratory, showing that patient ANG16A has no other exons deletions. (A) The electropherograms show results referring to patient ANG16A and controls DNA: (i) ANG25A_DEL, AS patient bearing a 4.5Mb heterozygous deletion comprising the whole UBE3A gene; (ii) InvDup06A Dup, a patient bearing a 15q11-q13 duplication; (iii) negative control (Control). (B) Graph displaying the ratios between the ANG16A patient's and controls' Relative Peak Areas (RPA) determined for the UBE3A gene. Reference values are as in the Figure 1.

sequence of exon 9 and introducing a premature termination signal at codon 27 (TGA) in this exon. If this mRNA is translated at all, the entire amino acid sequence of the HECT domain (last 350 aa) would be lost. This domain is very important for the function of UBE3A, that is, for ubiquitination and consequent degradation of target proteins (Huibregtse et al., 1993), potentially leading to an abnormal UBE3A transcript. The mother shows no symptoms of AS suggesting she carries the deletion allele on her paternal inactive 15q11-q13 region. These findings are consistent with the inheritance of a causative UBE3A deletion and confirm the diagnosis of AS in the sibling. Patient ANG16A as the other patients bearing mutations in the UBE3A gene mutations, display a typical intermediate phenotype compared to patients bearing large deletion cases of approximately 4-6 
A

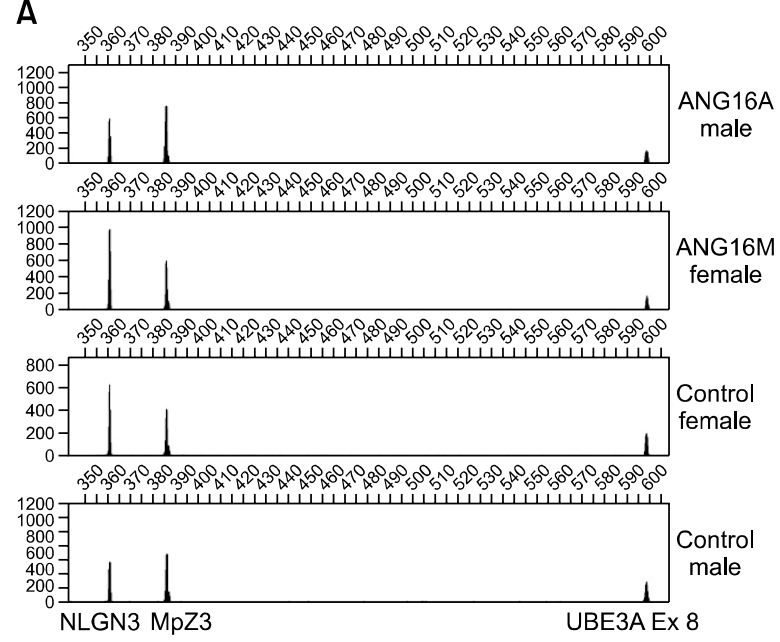

B

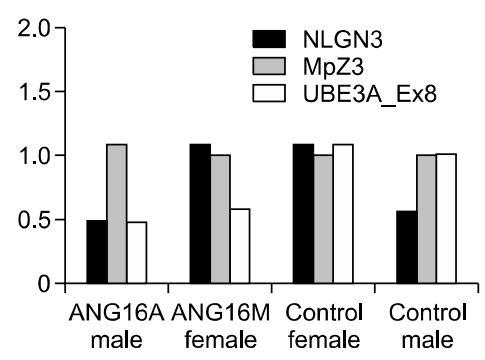

Figure 3. CMDA gene dosage analysis performed on patient ANG16A bearing a deletion of exon 8 of the UBE3A gene. (A) Electropherogram showing gene dosage variation for exon 8 among the patient, his mother and a male and female controls tested for NLGN3 (X-linked) and MpZ3 (autosomal) genes. (B) Histogram displaying the ratios between the ANG16A patient's and control's Relative Peak Areas (RPA) determined for the exon 8 of the UBE3A gene (see text for explanation). Reference values are as follows: RPA Ratio $\sim 1=$ normal dosage; RPA Ratio $\sim 0.5$ $=$ Heterozygous deletion. The values in UBE3A patients was confirmed in ten repeated experiments (mean RPA $=0.487$; SE \pm 0.052 ). In contrast, the mean RPA values determined from a series of DNA from twenty unrelated individuals is 1.126 (SE \pm 0.071 ). The sensitivity of this test to detect deletion/duplication was also verified by comparing the Relative Peak Areas between ten males (mean RPA $=0.565 ; \mathrm{SE} \pm 0.022$ ) and ten females (mean RPA $=1.112 ; \mathrm{SE} \pm 0.028$ ) using a X-linked NLGN3 gene as control.

$\mathrm{Mb}$, but have similar propensity to seizures and microcephaly (Varela et al., 2004).

To our knowledge, this is the first published work using the MLPA ME28-B1 and P336-A1 kits (MRC-Holland, Amsterdam, The Netherlands) to genotype the UBE3A gene in AS patients. A similar semi-quantitative gene-dosage method, previously described by Boyes et al. (2006), was aimed at analyzing only four of the ten UBE3A coding gene exons. Instead, the method proposed in this report is able to detect small deletions/duplications that might involve any of the UBE3A's exons as demonstrated in the patient we have studied. MLPA is a inexpensive, fast and accurate test to

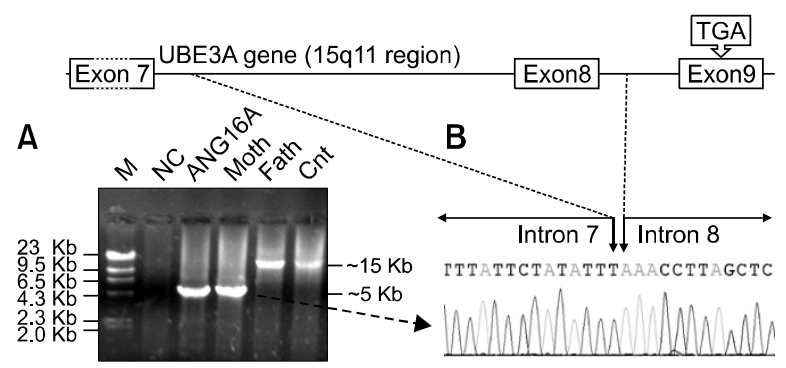

Figure 4. Breakpoint analysis of exon 8 deletion of the UBE3A gene in the ANG16 family: (A) Agarose gel electrophoresis of the long-range PCR products showing a normal amplicon of $\mathrm{ca} .15 \mathrm{~kb}$ in the father (Fath) and in a Control sample (Cnt), and a shorter $5 \mathrm{~kb}$ product in the AS patient (ANG16A) and his mother (Moth). NC $=$ Negative controls detecting DNA contamination. (B) Sequence analysis next to the breakpoints is consistent with a deletion of $9495 \mathrm{bp}$ spanning from intron 7 to intron 8, including exon 8 . This deletion introduces a frameshift and a premature stop codon (TGA) in exon 9 of the UBE3A gene.

detect gene dosage changes in a small but important proportion of AS cases of unidentifiable cause.

In conclusion we propose the use of MLPA tests (ME028-B1, P336-A1 from MRC-Holland and/or the MLPA test developed in our laboratory) to analyze UBE3A gene exons, in all patients and their relatives in the case their mutant genotypes remained undetermined after methylation and DNA sequencing analyses. ME028 is the primary MLPA kit for PWS/Angelman syndrome, detecting both copy number changes and imprinting defects of the $15 q 11$ region. We suggest that the P336 UBE3A MLPA probemix be useful as complementary diagnostic tool when the gene dosage analysis should be extended to the remaining coding exons. This strategy shall lead to the identification of novel mutant alleles and to a better genetic counselling in familial cases of AS.

\section{Methods}

\section{Case report}

The proband is a 7-year-7-month-boy born from no consanguineous parents. A first-degree cousin of his father had mental retardation. He was born at term, by normal delivery, after a normal gestation. Birth weight was $3,370 \mathrm{~g}$; birth length and cranial circumference are unknown. No perinatal and neonatal risk factors were reported. Psychomotor development was delayed. He held his head up at 8 months, reached the standing position at 18 months, walked with support at 24 months, vocalized at approximately 18 months, pronounced only two words at 24 months, and no other words were subsequently spoken, never reached the sphincter control. He came to our observation when he was 6-year-3-month old. Phenotypic features included microcephaly (cranial circumference 46.5 
$\mathrm{cm}$ ), beaked nose, small and flat philtrum, high palate, short fifth fingers of the hands, finger webbing, incompletely descended testes. After neurological examination, he presented severe mental retardation, drooling, paroxysmal laughter, generalized muscle hypotonia, hyperexcitable deep tendon reflexes at the four limbs, ataxia. $\mathrm{He}$ also presented scoliosis and flat-valgus foot. EEG showed high voltage spike-and-wave complexes over the parieto-temporo-occipital regions bilaterally, more prominent over the right hemisphere. Brain MRI revealed a mild posterior periventricular signal hyperintensity of the white matter and corpus callosum hypoplasia, more evident between body and splenium. At that time parents did not reported seizures.

\section{Methylation analysis}

Genomic DNA was isolated from peripheral blood lymphocytes using the salt chloroform extraction method. All DNA was quantified by spectrophotometry (Nanodrop) and checked for degradation on an agarose gel. Methylation analysis of the Prader-Willi/Angelman region of chromosome 15 , was performed by methylation-specific polymerase chain reaction at the SNRPN locus (MS-PCR), according to Zeschnigk et al. (1997), after deamination of cytosine to uracil by treatment with sodium bisulfite. Absence of a methylated maternal band and presence of the unmethylated paternal allele confirmed the diagnosis of AS.

\section{Sequence analysis of the UBE3A gene}

DNA sequence analysis included all coding UBE3A gene exons (Russo et al., 2000). All DNA samples were sequenced bidirectionally using the $\mathrm{ABI}$ Prism Big Dye Terminator Cycle Sequencing Ready Reaction Kit and the ABI 3130 Genetic analyzer (Applied Biosystems, Foster City, CA). DNA fragments amplified from genomic DNA were purified using Exonuclease I and Shrimp Alkaline Phosphatase according to the manufacturer's protocol (ExoSAP kit Amersham Biosciences, Uppsala, Sweden).

\section{MLPA analysis}

MLPA analysis was performed using the SALSA MLPA KIT ME028-B1 (MRC-Holland, Amsterdam, The Netherlands). The kit contains probes specific for the $15 q 11-13$ region, and some of these probes contain a Hhal recognition site that provide information about the methylation status. Besides the detection of aberrant methylation, many probes provide information on DNA copy number changes. In addition, different UBE3A gene exons (exons \# 1, 5, 6, 7, 8 and 13) probes are present. Exons numbering for the UBE3A gene is as described in the MLPA kit (MRCHolland, Amsterdam, The Netherlands). It is worth noting here that the numbering system used by the MRC-Holland might be different as compared to the literature. For the analysis of the remaining coding exons we have developed a in-house built MLPA assay using probes for coding exons \# 9, 10, 11, 12 and 14. Three additional probes hybridizing on other chromosomes, were also used as "control probes". Probes' sequences are shown in the Supplemental Data Table S1. These probes were designed as described in the "Synthetic probe design" protocol available at the MRC-Holland site (http://www.mlpa.com). Blast analysis for the probes' sequences, was also performed using the BLAST software (http://www.ncbi. nlm.nih.gov/BLAST/) to confirm that the selected sequences did not cross-hybridize with UBE3A pseudogenes located on chromosomes 2 and 21 (Kishino and Wagstaff, 1998).

MLPA analysis was carried out essentially as described by Schouten et al. (2002) and Calì et al. (2010). PCR products were identified and quantified by capillary electrophoresis on an $\mathrm{ABI} 3130$ genetic analyzer, using the Gene Mapper software from Applied Biosystems, Foster City, CA. In order to process efficiently the MLPA deletion/duplication data, a spreadsheet was generated in Microsoft Excel. First, the data corresponding to each sample (patient's and control's DNAs) were normalised by dividing each probe's signal strength (i.e., the area of each peak) by the average signal strength yielded by the 10 control probes to generate for each peak a Relative Peak Area (RPA) value. The RPA value for each probe in the patient's sample was then compared to that of a control's sample by dividing, for each peak, the patient's RPA by the control's RPA. The latter ratio was then used to define the following categories: (i) $\sim 1$, for the non-deleted/non-duplicated gene region, (ii) $\sim 0,5$ if deleted, (iii) $\sim 1,5$ if duplicated. Other detail on normalizing and quality test are as described in Calì et al. (2010).

\section{Comparative multiplex dosage analysis (CMDA)}

CMDA was performed as described in Rowland et al. (2001), Cali et al. (2010) and Di Bella et al. (2006) to confirm the deletion of the exon 8 of the UBE3A gene detected by MLPA. Briefly, exon 8 was co-amplified in a fluorescent multiplex PCR with Myelin protein zero (MpZ3) and Neuroligin 3 (NLGN3) control genes. A total of 10-15 pmol of each primer (sequencing and PCR conditions are available from the Authors on request), and 18 PCR cycles were used to ensure the reaction was kept within the exponential phase. The PCR products were resolved on ABI3130 (Applied Biosystems) sequence analyser according to manufacture's instructions. Results from the test sample (ANG16A patient) were compared separately with those from twenty (10 males and 10 females) non-deleted, non-duplicated control individuals. The Relative Peak Areas for each test were calculated as described above for MLPA. The sensitivity of this test to detect deletion/duplication was also verified by comparing the Relative Peak Areas (RPA) between males (RPA 0.5) and females (RPA 1) using a X-linked NLGN3 gene as control. A total of ten RPA values were calculated separately for patient ANG16A. The result of RPA was: (i) $\sim 1$, for the nondeleted/non-duplicated gene region tested in the patient, (ii) $\sim 0,5$ if deleted, (iii) $\sim 1,5$ if duplicated. Standard Deviation of the mean of ten RPA values obtained for patient ANG16A, and controls was calculated.

\section{Gap-PCR and sequence analysis}

The breakpoints were amplified by Long-range PCR performed using Expand Long Template PCR System 
(Roche) following the manufacturers' recommendations Briefly, $500 \mathrm{ng}$ of genomic DNA was subjected to PCR amplification in $50 \mu \mathrm{l}$ reaction volume containing the enzyme buffer $1 \times, 500 \mu \mathrm{M}$ of dNTPs, $300 \mathrm{nM}$ of each primer and 3 units of polymerase. PCR reaction as follows: initial denaturation of $94^{\circ} \mathrm{C}$ for $2 \mathrm{~min}, 30$ cycles of $94^{\circ} \mathrm{C}$ for $10 \mathrm{~s}, 55^{\circ} \mathrm{C}$ for $30 \mathrm{~s}$, final extension of $68^{\circ} \mathrm{C}$ for $10 \mathrm{~min}, 20 \mathrm{~s}$ of cycle elongation was applied in each of the first 10 cycles; soak at $4^{\circ} \mathrm{C}$. Primer sequences were designed on the intronic sequences (introns 6 and 9) of the UBE3A gene. The PCR product was analyzed on agarose gel and then directly sequenced on an ABI PRISM 310 sequencer by a primer walking strategy and compared with the reference sequence (Homo sapiens chromosome 15, Reference Sequence NC_000015.9)

\section{Acknowledgments}

This work was supported by the following fund of the Italian Ministry of Health: Ricerca Corrente 2009 entitled "Ritardo mentale, epilessia e autismo: studio genetico, clinico e neurofisiologico". Mirella Vinci is a Ph. student of the "Dottorato di Ricerca in Genomica e Proteomica nella Ricerca Oncologica ed Endocrino-Metabolica" of the University of Palermo.

\section{Supplemental data}

Supplemental Data include a table and can be found with this article online at http://e-emm.or.kr/article/article_files/ SP-42-12-06.pdf.

\section{References}

Abaied L, Trabelsi M, Chaabouni M, Kharrat M, Kraoua L, M'rad R, Tebib N, Maazoul F, Chaabouni H. A novel UBE3A truncating mutation in large Tunisian Angelman syndrome pedigree. Am J Med Genet A 2010;152A:141-6

Ainsworth PJ, Koscinski D, Fraser BP, Stuart JA. Family cancer histories predictive of a high risk of hereditary non-polyposis colorectal cancer associate significantly with a genomic rearrangement in $\mathrm{hMSH} 2$ or hMLH1. Clin Genet 2004;66:183-8

Boyes L, Wallace AJ, Krajewska-Walasek M, Chrzanowska $\mathrm{KH}$, Clayton-Smith J, Ramsden S. Detection of a deletion of exons 8-16 of the UBE3A gene in familial Angelman syndrome using a semi-quantitative dosage PCR based assay. Eur J Med Genet 2006;49:472-480

Burger J, Horn D, Tonnies H, Neitzel H, Reis A. Familial interstitial $570 \mathrm{kbp}$ deletion of the UBE3A gene region causing Angelman syndrome but not Prader-Willi syndrome. Am J Med Genet 2002;111:233-7

Calì F, Ruggeri G, Vinci M, Meli C, Carducci C, Leuzzi L, Pozzessere S, Schinocca P, Ragalmuto A, Chiavetta V, Miccichè $S$, Romano V. Exon deletions of the phenylalanine hydroxylase gene in Italian hyperphenylalaninemics. Exp Mol Med 2010;42:81-6

Clayton-Smith J, Laan L. Angelman syndrome: A review of the clinical and genetic aspects. J Med Genet 2003;40:87-95

Di Bella MA, Calì F, Seidita G, Mirisola M, Ragusa A, Ragalmuto A, Galesi O, Elia M, Greco D, Zingale M, Gambino G, D'Anna RP, Regan R, Carbone MC, Gallo A, Romano V. Screening of subtelomeric rearrangements in autistic disorder: identification of a partial trisomy of $13 q 34$ in a patient bearing a 13q;21p translocation. Am J Med Genet B Neuropsychiatr Genet 2006;141B:584-90

Fridman C, Varela MC, Kok F, Diament A, Koiffman CP. Paternal UPD15: further genetic and clinical studies in four Angelman syndrome patients. Am J Med Genet 2000; 92:322-7

Haverfield EV, Whited AJ, Petras KS, Dobyns WB, Das S. Intragenic deletions and duplications of the LIS1 and DCX genes: a major disease-causing mechanism in lissencephaly and subcortical band heterotopia. Eur J Hum Genet 2009;17:911-8

Huibregtse JM, Scheffner M, Howley PM. Localization of the E6-AP regions that direct human papillomavirus E6 binding, association with p53, and ubiquitination of associated proteins. Mol Cell Biol 1993;13:4918-27

Jiang YH, Lev-Lehman E, Bressler J, Tsai TF, Beaudet AL. Genetics of Angelman syndrome. Am J Hum Genet 1999;65:1-6

Kishino T, Lalande M, Wagstaff J. UBE3A/E6-AP mutations cause Angelman syndrome. Nat Genet 1997;15:70-3

Kishino T, Wagstaff J. Genomic organization of the UBE3A/ E6-AP gene and related pseudogenes. Genomics 1998; 47:101-7

Madrigal I, Rodríguez-Revenga L, Badenas C, Sánchez A, Martinez F, Fernandez I, Fernández-Buriel M, Milà M. MLPA as first screening method for the detection of microduplications and microdeletions in patients with $\mathrm{X}$-linked mental retardation. Genet Med 2007;9:117-22

Matsuura T, Sutcliffe JS, Fang P, Galjaard RJ, Jiang YH, Benton CS, Rommens JM, Beaudet AL. De novo truncating mutations in E6-AP ubiquitin-protein ligase gene (UBE3A) in Angelman syndrome. Nat Genet 1997;15:74-7

Moncla A, Malzac P, Voelckel MA, Auquier P, Girardot L, Mattei MG, Philip N, Mattei JF, Lalande M, Livet MO. Phenotype-genotype correlation in 20 deletion and 20 non-deletion Angelman syndrome patients. Eur $\mathrm{J}$ Hum Genet 1999;7:131-9

Petersen MB, Brondum-Nielsen K, Hansen LK, Wulff K. Clinical, cytogenetic, and molecular diagnosis of Angelman syndrome: Estimated prevalence rate in a Danish county. Am J Med Genet 1995;60:261-2

Priolo $M$, Sparago A, Mammì C, Cerrato F, Laganà C, Riccio A. MS-MLPA is a specific and sensitive technique for detecting all chromosome 11p15.5 imprinting defects of BWS and SRS in a single-tube experiment. Eur J Hum Genet 2008;16:565-71

Robb SA, Pohl KR, Baraitser M, Wilson J, Brett EM. The 'happy puppet' syndrome of Angelman: review of the clinical features. Arch Dis Child 1989;64:83-6

Rougeulle C, Glatt H, Lalande M. The Angelman syndrome 
candidate gene, UBE3A/E6-AP, is imprinted in brain. Nat Genet 1997; $17: 14-5$

Rowland JS, Barton DE, Taylor GR,UK Clinical Molecular Genetics Society HMSN Project Group. A comparison of methods for gene dosage analysis in HMSN type 1. J Med Genet 2001;38:90-5

Russo S, Cogliati F, Viri M, Cavalleri F, Selicorni A, Turolla L, Belli S, Romeo A, Larizza L. Novel Mutations of Ubiquitin Protein Ligase 3A Gene in Italian Patients with Angelman Syndrome. Hum Mutat 2000;15:387

Schouten JP, McElgunn CJ, Waaijer R, Zwijnenburg D, Diepvens F, Pals $G$. Relative quantification of 40 nucleic acid sequences by multiplex ligation-dependent probe amplification. Nucleic Acids Res 2002;30:e57
Varela MC, Kok F, Otto PA, Koiffmann CP. Phenotypic variability in Angelman syndrome: comparison among different deletion classes and between deletion and UPD subjects. Eur J Hum Genet 2004;12:987-92

Williams CA, Angelman $\mathrm{H}$, Clayton-Smith J, Driscoll DJ, Hendrickson JE, Knoll JH, Magenis RE, Schinzel A, Wagstaff $J$, Whidden EM. Angelman syndrome: consensus for diagnostic criteria. Angelman syndrome Foundation. Am J Med Genet 1995;56:237-8

Zeschnigk M, Lich C, Buiting K, Doerfler W, Horsthemke B. A single-tube PCR test for the diagnosis of Angelman and Prader-Willi syndrome based on allelic methylation differences at the SNRPN locus. Eur J Hum Genet 1997;5:94-8 